\title{
DEFORMAÇÕES INDUZIDAS POR PROCESSOS DE REBITAGEM
}

\author{
JOSÉ A. N. FIGUEIRA, DANIELLA Y. NEGRONI, LUÍS GONZAGA TRABASSO
}

\author{
Divisão de Engenharia Mecânica/Instituto Tecnológico de Aeronáutica \\ DCTA/ITA/IEM, Pça. Mal. Eduardo Gomes, $n^{\circ}$ 50. CEP 12228-900 São José dos Campos - SP \\ E-mails:jose.augusto.figueira@gmail.com,dani_yada@yahoo.com.br,gonzaga@ita.br
}

\begin{abstract}
Automated and manual riveting are usual assembly processes in aerospace metallic structures manufacturing such as fuselage panels and wing panels. During the structural riveting, deformations are generated due to large plastic displacements between rivets and the aircraft structural parts being attached. That problem is pronounced when dealing with large structures such as large fuselage or wing panels. This fact may result in geometrical penalties on the assembly of the segments, and, by consequence, the manufacturing team is usually forced to spend production hours to bypass the assembly difficulties generated by the riveting process. The final result may be a geometrically deformed segment detectable by metrology measurements (laser tracker measurements, or photogrammetry for example). This work is the continuation of a previous study aimed to identify a methodology to determine the induced deformations generated by the riveting process. Here an already accomplished exploratory previous factorial experiment $2^{5}$, with 5 repetitions, executed in 2005 , is assessed to identify the major influence contributor factors. The final aim is to gain a deeper understanding of the characteristic influence of each factor.
\end{abstract}

Keywords_ Systems for automated airframe manufacturing, riveting process, Induced deformation, Industrial Metrology

Resumo-Em processos de montagem de estruturas metálicas para uso aeronáutico, tais como painéis de fuselagem, painéis de asa, os processos de rebitagem automática e manual são os mais utilizados. Durante esses processos, deformações são induzidas devido à interação entre as deformações plásticas dos rebites e a estrutura circundante em processo de montagem. Esse problema é ainda mais perceptível em grandes estruturas como grandes painéis de fuselagem ou de asa. Este fato pode resultar em alterações geométricas dos segmentos estruturais e, por consequência, um tempo considerável é posteriormente exigido dos times de manufatura para contornar o problema gerado na rebitagem. O resultado final pode ser um segmento geometricamente deformado, suas deformações podem ser detectadas por processos de metrologia industrial, como o laser tracker e a fotogrametria, por exemplo. Este trabalho é continuação de um estudo anterior visando uma metodologia de determinação das deformações induzidas geradas pelo processo de rebitagem. O que aqui apresentamos são análises dos resultados de um primeiro experimento exploratório, um fatorial completo $2^{5}$, com 5 repetições, executado em 2005, visando identificar os principais fatores de influência sobre a variável medida. O objetivo final é aprofundar a compreensão da influência de cada fator de maior relevância.

Palavras-chave — Sistemas para manufatura aeronáutica automatizada, processos de rebitagem, deformações induzidas, metrologia industrial.

\section{Introdução}

$\mathrm{Na}$ indústria aeronáutica, processos de rebitagem (também denominados de processos de cravação) são amplamente usados nos procedimentos de montagem estrutural de painéis visando a formação de segmentos para posterior junção, por exemplo, para formação uma fuselagem (vide Figura1). Nesses processos de rebitagem pode ocorrer um fenômeno de deformação, ou distorção da estrutura, consequência da própria rebitagem. Esse fenômeno de deformação induzida e residual pode ser detectado por metrologia industrial, algumas vezes até mesmo visualmente sob forma de folgas ou degraus de montagem exageradamente acima dos valores previstos em projeto. Na literatura acadêmica existe muito pouco a respeito, também não há uma indicação, ou uma metodologia de previsão do fenômeno, um meio de antecipação dos seus efeitos, ou de minimizá-los.

O presente trabalho se baseia em dois trabalhos anteriores sobre o mesmo tema, com dados experimentais coletados e analisados, visando identificar os fatores influentes sobre a variável deformação indu- zida, em função dos parâmetros do processo de rebitagem, tais como: diâmetro de rebite, passo entre prendedores, espessura de pacote, força aplicada para conformação do rebite, materiais dos rebites e das estruturas, sequência de rebitagem, tipo de cabeça do rebite, folga/interferência entre furo e prendedor, etc. São muitos os fatores potencialmente influentes, necessário se faz identificar os mais significativos para o efeito em foco.

Com finalidade meramente ilustrativa, a Figura 2 mostra, esquematicamente, a deformação induzida típica de um painel de fuselagem de seção circular. Nela visualizamos o mesmo painel antes de depois de um processo de rebitagem. A deformação é percebida sob a forma de um aparente fechamento, ou seja, uma alteração local do raio de curvatura do painel. 


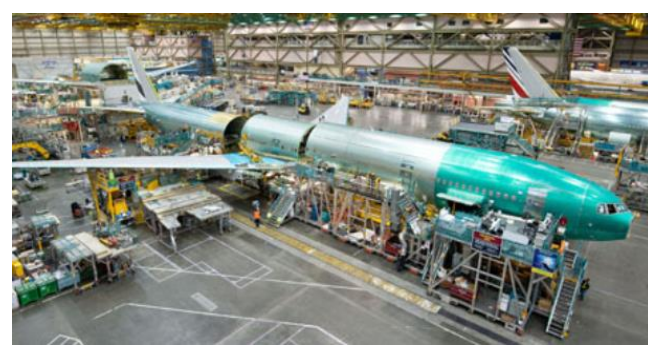

Fonte: http://boeing. mediaroom.com/index.php?s=13\&cat=26\&page=2\&item=1848

Figura1. Junção de segmentos de fuselagem.

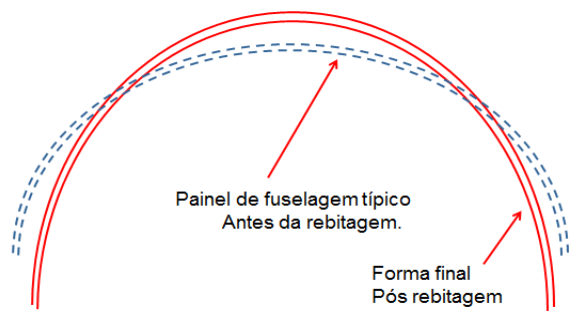

Figura 2. "Deformação" dos painéis após rebitagem.

Esse fechamento é o resultado de um componente de momento fletor gerado pela conjugação de uma expansão induzida pelos rebites inseridos no revestimento externo (vide Figuras 3 e 4) e uma reação de resistência nos elementos de reforço do painel, particularmente cavernas (vide Figura 5). Esse efeito conjugado expansão do revestimento e a resistência das cavernas gera esse fechamento, por vezes também um empenamento, ou também uma torção do mesmo (mais típico em painéis de asa).

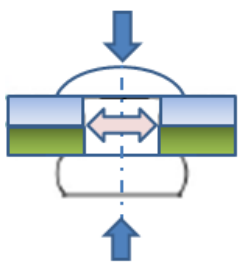

Figura 3. Conformação do rebite e expansão diametral do mesmo.

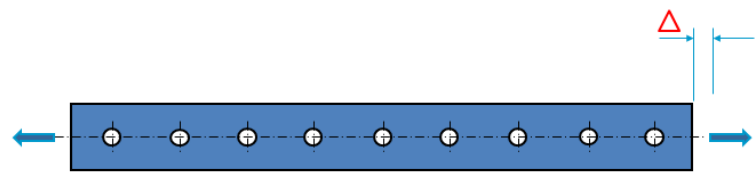

Figura 4. Expansão da linha de rebitagem por efeito da expansão diametral dos rebites.

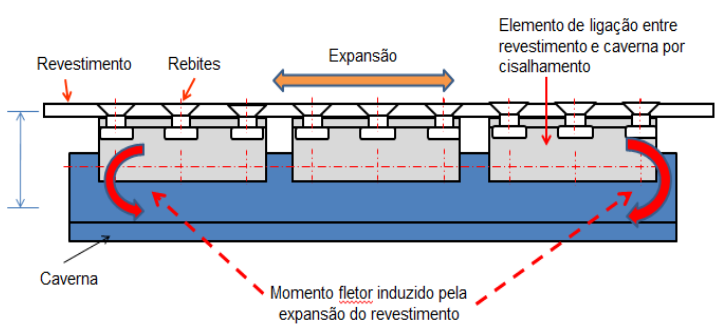

Figura 5. Momento fletor induzido pela expansão do revestimento
Como primeiro passo para análise desse fenômeno, a caracterização do processo de expansão do revestimento induzido pelos rebites nele inseridos era necessária. Um primeiro experimento exploratório foi proposto visando evidenciar a deformação, ou seja, a expansão das linhas de rebitagem.

Nesse primeiro experimento foram inicialmente selecionados 5 (cinco) fatores e avaliados sob forma de um projeto de experimento fatorial completo $2^{5}$, com 5 repetições, gerando um total de 160 medições em corpos de prova. Os resultados, base para a publicação da referência [1], evidenciaram a expansão da linha de rebitagem. Eles também mostraram que alguns dos fatores poderiam ser mais relevantes do que outros, embora sem especificamente adentrar mais detalhadamente nessa avaliação.

Neste trabalho foram retomados os mesmos dados obtidos anteriormente que foram submetidos a uma análise mais detalhada visando a identificação dos fatores de maior influência dentre os 5 (cinco) inicialmente escolhidos.

\section{Parte experimental}

\subsection{Descrição do experimento}

No experimento da referência [1], 160 cdps foram codificados, numerados e medidos antes e depois de uma rebitagem sequiencial de 12 rebites tipo Briles com diâmetro nominal de 5/32. A Figura 6 apresenta a configuração desses corpos de prova (cdps).

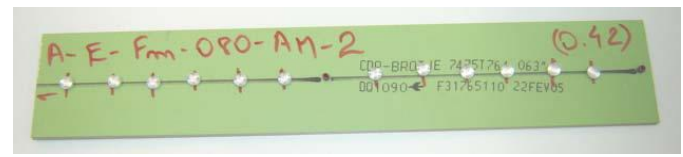

Figura 6: Corpos de prova de rebitagem utilizados, conforme referências [1] e [2].

Os 160 corpos de prova receberam uma codificação que identificava a configuração de cada um. A diferenciação de cada corpo de prova provinha da modificação de um ou mais dos 5 fatores selecionados para estudo e estão listados na Tabela 1. A Tabela 2 resume o resultado de todo o experimento e é a base para o presente trabalho.

Tabela 1: Variáveis re-analisadas neste trabalho a partir do primeiro experimento da referência [1].

\begin{tabular}{|c|c|c|c|c|c|c|}
\hline Fator & Identif. & Niveis & Codif. CDP & Descrição & Valor & Unidade \\
\hline Tipo de cravação & $\mathrm{A}$ & -1 & $\mathrm{~A}$ & Automática & $\mathrm{N} / \mathrm{A}$ & $\mathrm{N} / \mathrm{A}$ \\
\cline { 3 - 8 } & 1 & $\mathrm{M}$ & Manual & $\mathrm{N} / \mathrm{A}$ & $\mathrm{N} / \mathrm{A}$ \\
\hline Material do rebite & $\mathrm{B}$ & -1 & $\mathrm{AD}$ & Liga AL 2117 & $\mathrm{N} / \mathrm{A}$ & $\mathrm{N} / \mathrm{A}$ \\
\hline & & 1 & $\mathrm{E}$ & Liga AL 7050 & $\mathrm{N} / \mathrm{A}$ & $\mathrm{N} / \mathrm{A}$ \\
\hline Diâmetro do furo & $\mathrm{C}$ & -1 & $\mathrm{Fm}$ & Furo de folga máxima & 4.09 & $\mathrm{~mm}$ \\
\hline & 1 & $\mathrm{FM}$ & Furo de folga minima & 4.24 & $\mathrm{~mm}$ \\
\hline $\begin{array}{c}\text { Espessura do pacote } \\
\text { cravado }\end{array}$ & $\mathrm{D}$ & -1 & 050 & Espessura minima & 2.8 & $\mathrm{~mm}$ \\
\hline Altura do rebite & $\mathrm{E}$ & -1 & 080 & Espessura máxima & 3.6 & $\mathrm{~mm}$ \\
\cline { 2 - 8 } & 1 & $\mathrm{Am}$ & Altura minima & Rebite 0.3 abaixo & $\mathrm{mm}$ \\
\hline
\end{tabular}


Tabela 2: Resultados experimento fatorial completo $2^{5}$ conforme referência [1].

\begin{tabular}{|c|c|c|c|c|c|c|c|c|c|c|c|}
\hline Ensaio & Código & $\overline{\mathbf{A}}$ & B & C & D & $E$ & $\mathrm{Y1}$ & Y2 & Y3 & $\begin{array}{l}\mathrm{Y} 4 \\
\end{array}$ & Y5 \\
\hline 1 & A-AD-Fm-050-Am & -1 & -1 & -1 & -1 & -1 & 0.06 & 0.05 & 0.04 & 0.05 & 0.07 \\
\hline 2 & A-AD-Fm-050-AM & -1 & -1 & -1 & -1 & 1 & 0.05 & 0.03 & 0.04 & 0.03 & 0.03 \\
\hline 3 & A-AD-Fm-080-Am & -1 & -1 & -1 & & -1 & 0.04 & 0.02 & 0.02 & 0.02 & 0.02 \\
\hline 4 & A-AD-Fm-080-AM & -1 & -1 & -1 & & 1 & 0.07 & 0.06 & 0.01 & 0.03 & 0.02 \\
\hline 5 & A-AD-FM-050-Am & -1 & -1 & & -1 & -1 & 0.07 & 0.05 & 0.06 & 0.11 & 0.05 \\
\hline 6 & A-AD-FM-050-AM & -1 & -1 & & & 1 & 0.05 & 0.04 & 0.02 & 0.03 & 0.03 \\
\hline 7 & A-AD-FM-080-Am & -1 & -1 & & & -1 & 0.03 & 0.03 & 0.04 & 0.03 & 0.02 \\
\hline 8 & A-AD-FM-080-AM & -1 & -1 & 1 & 1 & 1 & 0.00 & 0.00 & 0.00 & 0.00 & 0.00 \\
\hline 9 & A-E-Fm-050-Am & -1 & 1 & 1 & & & 0.06 & 0.06 & 0.05 & 0.05 & \\
\hline$\frac{5}{10}$ & E-Fm-050-AM & -1 & $\frac{1}{1}$ & -1 & & 1 & 0.04 & 0.04 & 0.03 & 0.01 & 0.03 \\
\hline 11 & A-E-Fm-080-Am & -1 & 1 & -1 & 1 & -1 & 0.02 & 0.04 & 0.02 & 0.01 & 0.06 \\
\hline 12 & $E-F m-1$ & -1 & 1 & & & 1 & 0.00 & 0.02 & 0.02 & 0.01 & 0.02 \\
\hline 13 & A-E-FM-050-Am & & 1 & & & -1 & 0.06 & 0.07 & 0.04 & 0.06 & 0.03 \\
\hline 14 & A-E-FM-050-AM & -1 & 1 & 1 & -1 & 1 & 0.06 & 0.05 & 0.04 & 0.05 & 0.06 \\
\hline 15 & A-E-FM-080-Am & -1 & 1 & 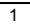 & 1 & -1 & 0.01 & 0.02 & 0.04 & 0.01 & 0.02 \\
\hline 16 & A-E-FM-080-AM & -1 & 1 & 1 & & 1 & 0.02 & 0.03 & 0.01 & 0.01 & 0.01 \\
\hline 17 & M-AD-Fm-050-Am & 1 & -1 & -1 & -1 & -1 & 0.02 & 0.04 & 0.05 & 0.04 & 0.06 \\
\hline 18 & M-AD-Fm-050-AM & 1 & -1 & -1 & -1 & 1 & 0.04 & 0.02 & 0.03 & 0.04 & 0.04 \\
\hline 19 & M-AD-Fm-080-Am & 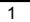 & -1 & -1 & & -1 & 0.05 & 0.02 & 0.01 & 0.01 & 0.01 \\
\hline 20 & $M-A D$ & 1 & -1 & -1 & & 1 & 0.04 & 0.02 & 0.02 & 0.04 & 0.03 \\
\hline 21 & M-AD-FM-050-Am & 1 & -1 & 1 & & -1 & 0.03 & 0.03 & 0.03 & 0.03 & 0.04 \\
\hline 22 & M-AD-FM-050-AM & 1 & -1 & 1 & -1 & 1 & 0.05 & 0.02 & 0.07 & 0.05 & 0.05 \\
\hline 23 & M-AD-FM-080-Am & 1 & -1 & 1 & 1 & -1 & 0.06 & 0.02 & 0.06 & 0.03 & 0.06 \\
\hline 24 & M-AD-FM-080-AM & 1 & -1 & 1 & 1 & 1 & 0.02 & 0.05 & 0.03 & 0.04 & \\
\hline 25 & M-E-Fm- & & 1 & & & -1 & 0.02 & 0.04 & 0.02 & 0.02 & 0.01 \\
\hline 26 & M-E-Fm-050-AM & 1 & 1 & -1 & -1 & 1 & 0.04 & 0.00 & 0.01 & -0.01 & 0.02 \\
\hline 27 & M-E-Fm-080-Am & 1 & 1 & -1 & 1 & -1 & 0.02 & 0.02 & 0.00 & 0.00 & 0.02 \\
\hline 28 & M-E-Fm-080-AM & & 1 & -1 & & 1 & -0.01 & -0.01 & 0.01 & -0.03 & -0.01 \\
\hline 29 & M-E-FM-050-Am & 1 & 1 & 1 & -1 & -1 & 0.03 & 0.02 & 0.06 & 0.04 & 0.03 \\
\hline 30 & M-E-FM-050-AM & & 1 & & & & 0.03 & 0.02 & 0.03 & 0.04 & 0.04 \\
\hline 31 & M-E-FM-C & 1 & 1 & 1 & 1 & -1 & 0.02 & 0.02 & 0.03 & 0.01 & 0.01 \\
\hline 32 & M-E-FM-080-AM & 1 & 1 & 1 & 1 & 1 & 0.00 & 0.00 & 0.02 & 0.00 & -0.01 \\
\hline
\end{tabular}

O mensurando, deformação induzida, é uma deformação linear medida após a rebitagem de cada cdp. Neste caso, é uma medição indireta, pois o mensurando é obtido por uma subtração:

$$
\mathrm{Yi}=\mathrm{Lf}-\mathrm{Li}
$$

em que:

Yi = deformação de rebitagem (mensurando);

$\mathrm{Lf}=$ Comprimento final do cdp (após rebitagem);

$\mathrm{Li}=$ Comprimento inicial do cdp (antes da rebitagem). Acrescentar unidades

O cálculo do mensurando, conforme Equação (1), tem a vantagem de anular os erros sistemáticos de medição eventualmente presentes na tomada de Lf e de Li, uma vez que o erro sistemático existente em cada medida é anulado, restando apenas a parcela dos erros aleatórios de cada medição.

No experimento original foi executada uma medição diferencial devido à maior confiabilidade que esta forma de medição apresenta. Para isso, um dos cdps fabricado foi marcado como cdp de referência e este cdp não foi rebitado. Em cada nova medição, o relógio comparador era zerado no cdp de referência, e todos os demais cdps foram medidos em relação a este, tanto antes, como depois do processo de rebitagem. Tal procedimento possibilitou ter uma medida física materializada para todos os cdps, o que contribuiu para redução da incerteza do resultado final, reduzindo a propagação de erros.

O modelo matemático sendo avaliado nesta reanálise do experimento é modelado por: $Y_{\mathrm{ijklmn}}=\mu+\alpha_{\mathrm{i}}+\beta_{\mathrm{j}}+\zeta_{\mathrm{k}}+\delta_{\mathrm{l}}+\xi_{\mathrm{m}}+(\boldsymbol{\alpha} \beta)_{\mathrm{ij}}+(\alpha \zeta)_{\mathrm{ik}}$
$+\ldots+(\alpha \beta \zeta)_{\mathrm{ijk}}+\ldots+\left(\alpha \beta \zeta \delta \xi_{\mathrm{ijklm}}+\mathrm{e}_{\mathrm{ijklmn}}\right.$ $\boldsymbol{\mu}=$ grande média;

$\boldsymbol{\alpha}_{i}=$ Influência do fator tipo de cravação;

$\boldsymbol{\beta}_{\mathbf{j}}=$ Influência do fator material do rebite;

$\zeta_{\mathbf{k}}=$ Influência do fator diâmetro do furo;

$\boldsymbol{\delta}_{\mathbf{l}}=$ Influência do fator espessura;

$\xi_{\mathrm{m}}=$ Influência do fator altura do rebite;

$(\boldsymbol{\alpha} \boldsymbol{\beta} . . .)_{\mathrm{ij} . . .}=$ Influência da interação entre os parâmetros dois a dois, três a três etc.;

$\mathbf{e}_{\mathrm{ijklmn}}=$ Erro aleatório;

\subsection{Análise dos resultados do experimento}

A Figura 7 mostra os gráficos tipo boxplot dos fatores e seus efeitos na variável deformação induzida.

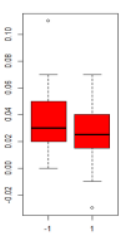

A: Processo: Auto x Man

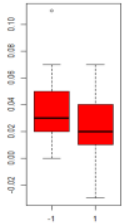

B: Material do Rebite

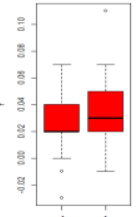

C: Diâmetro do Furo

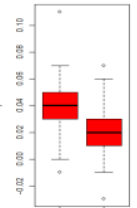

D: Espessura do Pacote

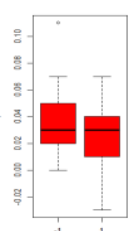

E: Altura do Rebite
Figura 7. Boxplot dos fatores selecionados

Os gráficos da Figura 7 aparentemente mostram que a espessura do pacote (fator D) é o fator de maior influência dentre os cinco avaliados. Uma análise de variância feita sobre os dados do experimento é detalhada a seguir.

\subsubsection{Relevância dos fatores}

Todos os cinco fatores selecionados são estatisticamente relevantes, mas a ordem de influência sobre a variável deformação induzida, do maior ao menor, com nível de significância de 5\%, ou menor (isto é, um nível de confiança de 95\%, ou superior) é a apresentada na Tabela 3, para a ANOVA dos fatores, obtida por meio do software $<\mathrm{R}\rangle$, ordenado por ordem decrescente de influência, verificada pelo parâmetro estatístico $F$-value:

É importante notar que os dez (10) fatores, ou interações, identificados como os de maior relevância, foram os seguintes:

1) Espessura do pacote cravado (D): Quanto maior a espessura, menor é a deformação. Isso é válido para ambas as formas de cravação: manual ou automática;

2) Material do rebite (B): O material mais mole induziu substancialmente maior deformação na rebitagem manual que o material mais duro. Na rebitagem automática, quase não houve diferença.

em que: 
3) A altura do rebite (E) é o terceiro fator em ordem de influência, com influência muito maior na rebitagem automática do que na manual.

4) A interação processo de cravação $x$ material do rebite $(\mathrm{AB})$, onde para o rebite mais duro a cravação manual causou a menor deformação.

5) O processo de cravação (A), se automático, ou manual, com maior influência no processo automático.

6) A interação tripla: material do rebite $\mathrm{x}$ diâmetro do furo $\mathrm{x}$ altura do rebite (BCE). A análise dessa interação deverá ser aprofundada num projeto de um próximo experimento.

7) A interação: processo de cravação x espessura do pacote cravado (AD), onde a combinação do processo automático com a menor espessura causou a maior deformação.

8) A interação: processo de cravação x diâmetro do furo (AC): Na cravação automática o furo de menor folga gerou a maior deformação, o que é coerente com as teorias de plasticidade. Na cravação manual ocorreu o oposto.

9) A interação tripla: diâmetro do furo $x$ espessura do pacote cravado $\mathrm{x}$ altura do rebite (CDE).

10) A interação quádrupla: Processo de Cravação x Material do Rebite x Diâmetro do furo x Altura do Rebite (ABCE). Surpreendentemente essa interação de alta ordem foi mais relevante que o próprio fator diâmetro do furo $(\mathrm{C})$ e que as demais interações duplas e triplas possíveis.

\subsubsection{Relevância das Interações entre Fatores}

É importante notar a relativamente alta influência das interações entre fatores. Interações duplas, triplas e quádruplas foram todas significantes no resultado do experimento, mais até que o fator diâmetro do furo (C) tomado isoladamente. Os resultados de deformação máxima e de deformação mínima ocorreram em efeitos extremos de interação. Assim, neste trabalho, a análise das interações é de fundamental importância para a compreensão do fenômeno como um todo.

A Figura 8 mostra os efeitos das interações dois a dois. Neste nível de interações, as mais significativas foram: processo de cravação x material do rebite; processo de cravação $\mathrm{x}$ espessura do pacote cravado; processo de cravação x diâmetro do furo; processo de cravação x altura do rebite.

Algumas interações podem ser explicadas com relativa facilidade pela suas próprias naturezas. É o caso da interação processo de cravação $\mathrm{x}$ material do rebite, gráfico (a) - Y A*B da Figura 8.
Tabela 3: Análise de Variância dos Fatores Selecionados

\begin{tabular}{|c|c|c|c|c|c|c|}
\hline Fator & Df & Sum Sq & Mean Sq & F-value & $\mathrm{pr}>\mathrm{F}$ & sig \\
\hline FD & 1 & 0.015602 & 0.015602 & 85.493 & $6.64 \mathrm{E}-16$ & $* * *$ \\
\hline FB & 1 & 0.00484 & 0.00484 & 26.521 & $9.59 \mathrm{E}-07$ & *** \\
\hline $\mathrm{FE}$ & 1 & 0.004 & 0.004 & 21.918 & 7.15E-06 & *** \\
\hline FA:FB & 1 & 0.003423 & 0.003423 & 18.753 & 2.98E-05 & *** \\
\hline FA & 1 & 0.00324 & 0.00324 & 17.753 & 4.71E-05 & *** \\
\hline FB:FC:FE & 1 & 0.001822 & 0.001822 & 9.986 & 0.00197 & ** \\
\hline FA:FD & 1 & 0.00169 & 0.00169 & 9.26 & 0.00284 & ** \\
\hline FA:FC & 1 & 0.00144 & 0.00144 & 7.89 & 0.00575 & ** \\
\hline FC:FD:FE & 1 & 0.00121 & 0.00121 & 6.63 & 0.01116 & * \\
\hline FA:FB:FC:FE & 1 & 0.00121 & 0.00121 & 6.63 & 0.01116 & * \\
\hline FA:FC:FD & 1 & 0.001 & 0.001 & 5.479 & 0.02079 & * \\
\hline FA:FB:FE & 1 & 0.001 & 0.001 & 5.479 & 0.02079 & * \\
\hline FC & 1 & 0.000902 & 0.000902 & 4.945 & 0.02792 & * \\
\hline FA:FD:FE & 1 & 0.000902 & 0.000902 & 4.945 & 0.02792 & * \\
\hline FA:FE & 1 & 0.000722 & 0.000722 & 3.959 & 0.04876 & * \\
\hline FB:FD & 1 & 0.00064 & 0.00064 & 3.507 & 0.0634 & \\
\hline FC:FD & 1 & 0.000562 & 0.000562 & 3.082 & 0.08155 & \\
\hline FA:FB:FC:FD & 1 & 0.000562 & 0.000562 & 3.082 & 0.08155 & \\
\hline FB:FC:FD:FE & 1 & 0.000563 & 0.000563 & 3.082 & 0.08155 & \\
\hline FB:FC & 1 & 0.00036 & 0.00036 & 1.973 & 0.16259 & \\
\hline FA:FB:FD & 1 & 0.000302 & 0.000302 & 1.658 & 0.20026 & \\
\hline FB:FD:FE & 1 & 0.000302 & 0.000302 & 1.658 & 0.20026 & \\
\hline FB:FE & 1 & 0.000203 & 0.000203 & 1.11 & 0.29416 & \\
\hline FA:FC:FE & 1 & 0.000202 & 0.000202 & 1.11 & 0.29416 & \\
\hline FA:FB:FC:FD:FE & 1 & 0.00016 & 0.00016 & 0.877 & 0.35087 & \\
\hline FB:FC:FD & 1 & 0.00009 & 0.00009 & 0.493 & 0.4838 & \\
\hline FA:FB:FD:FE & 1 & 0.00004 & 0.00004 & 0.219 & 0.64046 & \\
\hline FA:FB:FC & 1 & 0.000023 & 0.000023 & 0.123 & 0.72607 & \\
\hline FC:FE & 1 & 0.00001 & 0.00001 & 0.055 & 0.8153 & \\
\hline FD:FE & 1 & 0.00001 & 0.00001 & 0.055 & 0.8153 & \\
\hline FA:FC:FD:FE & 1 & 0.000003 & 0.000003 & 0.014 & 0.90701 & \\
\hline Residuals & 128 & 0.02336 & 0.000183 & & & \\
\hline
\end{tabular}

Na cravação automática a influência do material do rebite é relativamente muito pequena, já na cravação manual a influência do resultado representa $100 \%$ de acréscimo em relação ao valor menor. Isso se dá pelo fato de, na cravação manual, o uso de rebites mais duros incomoda sobremaneira $\mathrm{O}$ operador, chega mesmo a causar dores nas mãos, o que faz com que o operador acione a máquina rebitadora (um martelete pneumático) o mínimo possível para a conformação do rebite. Logo, a deformação plástica do rebite mais duro é bem menor no processo manual que no automático. Essa diferença pode também explicar outras interações como o material do rebite $\mathrm{x}$ diâmetro do furo: essa interação está mascarada pelo processo de cravação. Outra interação mascarada é o processo de cravação $\mathrm{x}$ altura do rebite: ela está mascarada pelo material do rebite interagindo com o processo manual.

O mesmo se pode dizer da interação material do rebite $\mathrm{x}$ diâmetro do furo. A conclusão é que o processo de cravação (automático x manual) deveria ser olhado como bloco e não como fator e as considerações e conclusões devem ser obtidas dentro de cada processo particular.

Outro fator que, surpreendentemente, não se mostrou significativo foi o diâmetro do furo (C). Pela teoria da plasticidade esse fator deveria ser tão, ou mais influente que o fator altura do rebite (E). No entanto os resultados não evidenciaram isso. Um dos motivos está possivelmente numa falha do processo de furação dos cdps. A distinção entre furo de menor folga e furo de maior folga foi feita pelo tipo de broca usada na furação: Todos os cdps classificados de furo mínimo foram furados com uma broca desgastada (teoricamente com furo próximo ao limite inferior que é de $4.09 \mathrm{~mm}$ ). Todos os cdps classificados de furo máximo foram furados com uma 
broca nova, isto é, teoricamente com furo próximo de $4.24 \mathrm{~mm}$. Na verdade nem as brocas nem os diâmetros dos furos foram efetivamente medidos nesse experimento. A própria afiação das brocas altera o diâmetro do furo resultante. Assim não temos efetiva certeza de que os dois tipos de furo eram realmente diferentes entre si no nível assumindo. Isso deve ser corrigido num próximo experimento.

(a) $\mathrm{A} \times \mathrm{B}$

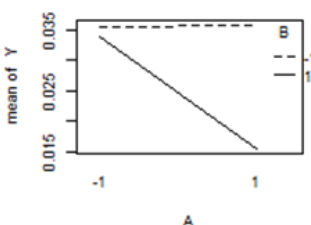

(c) $\mathrm{A} \times \mathrm{D}$

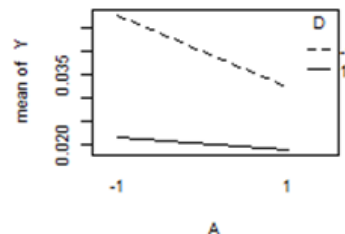

(e) $\mathrm{B} \times \mathrm{C}$
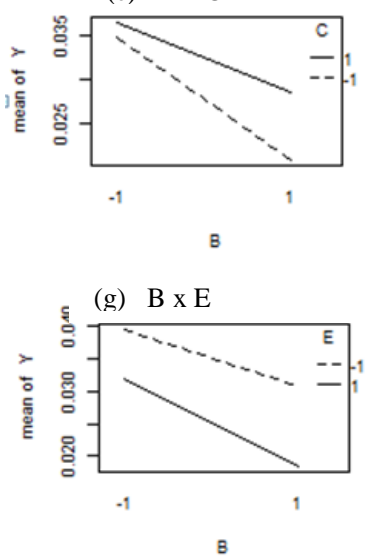

(i) $\mathrm{C} \times \mathrm{E}$

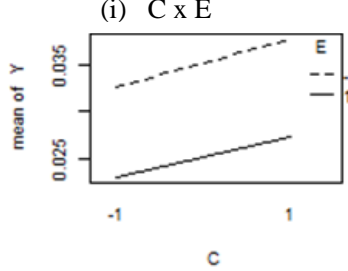

Figura 8. Interação entre os fatores (dois a dois).

\subsubsection{Análise dos resíduos}

Os resíduos se mostraram muito altos quando comparados à variável medida. A Figura 9 mostra uma análise da normalidade dos resíduos e uma visualização da distribuição dos mesmos. O gráfico mostra que, para um intervalo entre \pm 2 (dois) desvios padrões da amostra, podemos assumir que os resíduos são normais.
A Figura 10 mostra a distribuição dos resíduos em função do valor medido da deformação induzida. Nota-se que há uma tendência dos resíduos se agruparem ao longo de uma linha diagonal partindo do canto inferior esquerdo e subindo para o canto superior direito do gráfico. Esse comportamento dos resíduos é um indício de que pode haver outro fator relevante não considerando neste experimento.

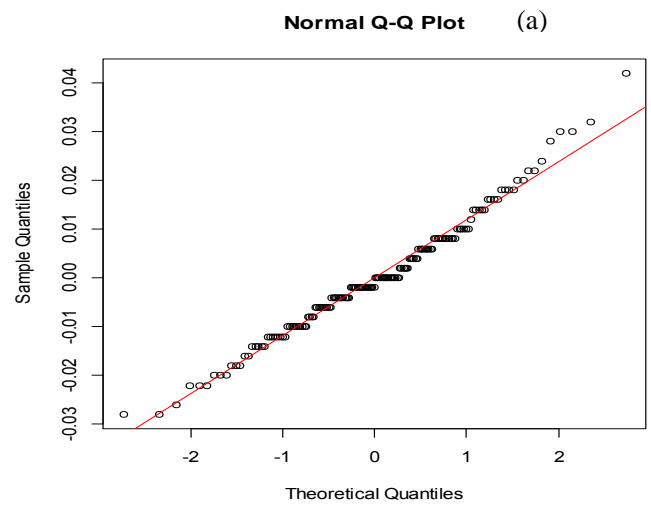

(b)

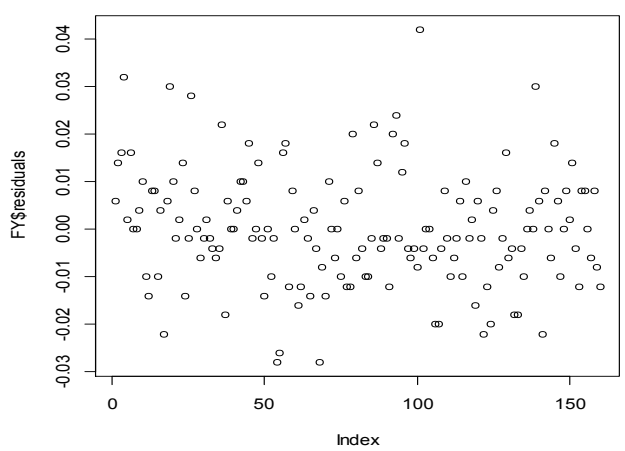

Figura 9. Análise da normalidade dos resíduos.

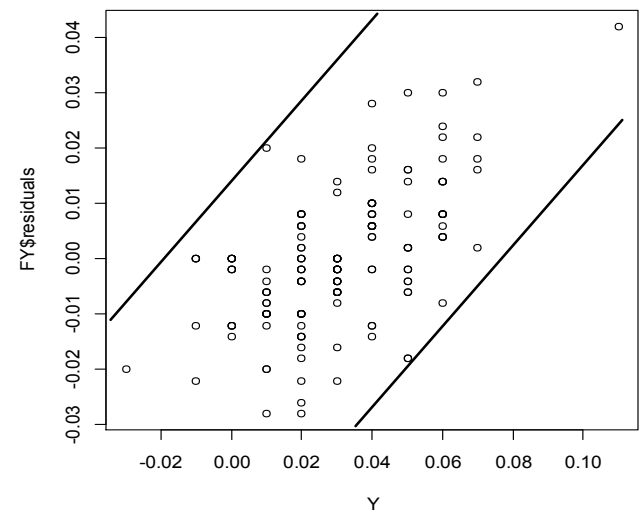

Figura 10. Resíduos em função da variável medida.

As referências [5] e [6] indicam que há outro fator, muito relevante nesse processo, mas não contemplado nesse primeiro experimento. Trata-se da força de conformação do rebite (na literatura indicado como squeezing force). Esse fator deve ser registrado, 
mas só é possível controlá-lo nos processos de cravação automática. Num processo de cravação manual não é possível fazê-lo diretamente. Isso reforça o fato de que os próximos experimentos devem contemplar apenas a cravação automática, mesmo porque ela é a mais utilizada na indústria. A rebitagem manual fica normalmente reservada para as áreas onde não há possibilidade de acesso para o processo automático.

\section{Conclusões e considerações finais}

Os fatores selecionados são todos relevantes para a indução da deformação, mas ainda há fatores potencialmente relevantes não avaliados. O nível de ruído de fundo (erros aleatórios) foram comparativamente muito grandes e é de fundamental importância projetar um novo experimento onde esses ruídos sejam reduzidos. A principal fonte de ruído parece ter sido o próprio mensurando e a resolução do sistema de medição. Assim um novo conceito de corpo de prova se faz necessário e o mesmo é proposto na Figura 11 para continuidade dos estudos. Esse novo corpo de prova possui 3(três) linhas de cravação com 30 rebites em cada uma; seis furos, dois a dois alinhados com cada linha de cravação, para medição dos comprimentos antes e após rebitagem. Cada cdp deve ser medido ao longo de cada linha (são três medições por cdp) e os resultados somados e divididos por 3. Igualmente o sistema de medição deverá ser capaz de ter uma resolução inferior a $0.01 \mathrm{~mm}$, o ideal seria uma resolução de $0.001 \mathrm{~mm}$ e uma incerteza de medição inferior a $0.003 \mathrm{~mm}$, o que é possível com o uso de uma máquina de medição por coordenadas (MMC).

Houve corpos de prova que empenaram bastante após a cravação, dificultando a medição, ou mesmo mascarando o resultado. Uma alternativa seria aplicar dispositivos de desempeno dos cdps visando minimizar esse efeito.

Os diâmetros dos furos devem ser efetivamente medidos e registrados. Um novo fator $<$ Força de conformação>, ou <Energia de deformação> deve ser adicionado e monitorado. Outro fator adicional é o passo entre rebites (pitch), que normalmente varia entre 4 e 6 vezes o diâmetro nominal do rebite. Outros fatores adicionais como uso de prendedores não deformáveis, tipo pinos Hilock ${ }^{\mathrm{TM}}$, Lockbolt $^{\mathrm{TM}}$, podem alterar bastante a variável deformação induzida e devem ser igualmente estudados. A forma e tipo de cabeça do prendedor pode também ser um fator relevante a ser avaliado. A direção de grão das chapas também deve ser um fator controlado, pois pode haver uma influência no movimento de discordâncias entre grãos, com propagação diferente na direção do grão em relação à direção ortogonal ao mesmo. Recomenda-se acrescentar a sequência de cravação como fator adicional do experimento, não obstante ser este fator é muito mais complexo por possuir uma vasta gama de possibilidades de implementação.
Uma proposta alternativa, de menor custo, é a simulação computacional desse fenômeno. Algumas iniciativas foram feitas nesse sentido (vide referências [3], [5] e [6]). Mas todas essas simulações devem inicialmente ser validadas por experimentos antes de ser possível assumir com confiabilidade os seus resultados.

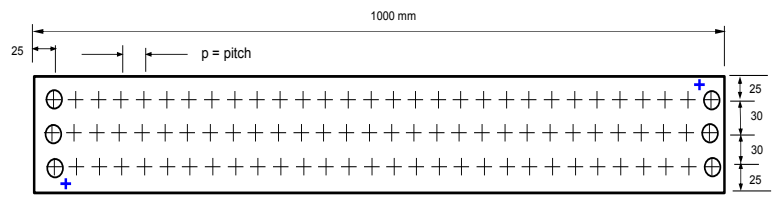

Figura 11. Proposição de um novo corpo de prova (cdp)

\section{Referências Bibliográficas}

[1] NEGRONI, D. Y. -“Deformação induzida pelo processo de rebitagem". Master Degree Thesis. ITA, 2006, 73p.

[2] NEGRONI, D. Y., TRABASSO, L. G. - A replicated two-level total factorial analysis: deformation in aluminum alloy skins caused by riveting processes. Product (IGDP), v. 9, p. 59-70, 2011.

[3] CUNHA, F. R., FIGUEIRA, J. A., BARROS, M.C.- "Methodology to Capture Induced Strains on Riveting Process of Aerospace Structures" 2010-36-0016I , SAE Brazil 2010. DOI: 10.4271/2010-36-0016

[4] ALBERTAZZI, A. G. Jr., SOUSA, A. R. "Fundamentos de Metrologia Científica e Industrial"- Barueri, SP, Manole,2008.

[5] LI, G.; SHI, G. "Effect Of The Riveting Process On The Residual Stress In Fuselage Lap Joints"50th AGM and Conference 16th Aerospace Structures and Materials Symposium. Montréal, 28-30 April 2003.

[6] MULLER, R.P.G. - An Experimental and Analytical Investigation on The Fatigue Behavior of Fuselage Riveted Lap Joints.- Doctor Degree Thesis. Delf University, 1995, 516p.

[7] MONTGOMERY, D. C.(2009). Design and Analysis of Experiments, $7^{\text {th }}$ edition, Wiley, New York 